\title{
UEBER EXTRAREGIONAERE GESCHLECHTSZELLEN BEI BRADYPUS
}

\author{
VON
}

\author{
H. M. DE BURLET
}

(Mit 5 Figuren im Text)

(Aus dem Anat. Institut der Universität Utrecht)

\section{EINLEITUNG}

Der Jubilar, dessen Festschrift diese Zeilen gewidmet sein sollen, hatte vor einer Reihe von Jahren die Liebenswürdigkeit mir einen jungen Embryo von Bradypus cuculliger zu überlassen, welcher sich seitdem als wertvolles Vergleichsobjekt schon in vieler. Hinsicht bewährt hat. Seine Nackensteisslänge betrug $17 \frac{1}{2} \mathrm{~mm}$; bei einer vorigen Gelegenheit wurde eine Abbildung von dem Embryo, in Seitenansicht, veröffentlicht ${ }^{1}$ ). Kopf, Rumpf und Extremitäten wurden gesondert zu Serien verarbeitet; die Fixierung erwies sich als vorzüglich. Heute möchte ich über die extraregionären Geschlechtszellen dieses seltenen Tieres einige Beobachtungen mitteilen.

Zur Orientierung über den Entwicklungsgrad des Urogenitalsystems im ganzen, sei auf die Abbildungen 1 und 2 verwiesen, wo man die gegenseitigen Lagebeziehungen seiner Bestandteile erkennt. In der ersten Figur ist linkerseits die Lage der Nebenniere, der definitiven Niere, und des Ureters wiedergegeben; rechts die Umgrenzung von Urniere und Geschlechtsdrüse, sowie der Verlauf des MüLLER'schen und WOLFF'schen Ganges. Die zweite, wie die erste graphisch reconstruierte, Zeichnung zeigt uns die selben Organe in der Projektion auf einer sagittalen Ebene. Die Urniere ist ein stattliches Organ, welches die dorsal und retroperitoneal gelegene definitive Niere von ventral verdeckt. Sie ist mittels einer Serosaduplicatur an die dorsale Rumpfwand befestigt (Urnierenligament). Die Geschlechtsdrüse hat sich als Plica genitalis von der Plica mesonephridica teilweise abgelöst und bleibt mit der Urniere ebenfalls durch eine Serosaduplicatur (mesorchium, mesovarium) verbunden; der obere Abschnitt hat dabei im vergleich zum Unteren grössere Selbständigkeit erlangt. Auf den Schnitten, welche das

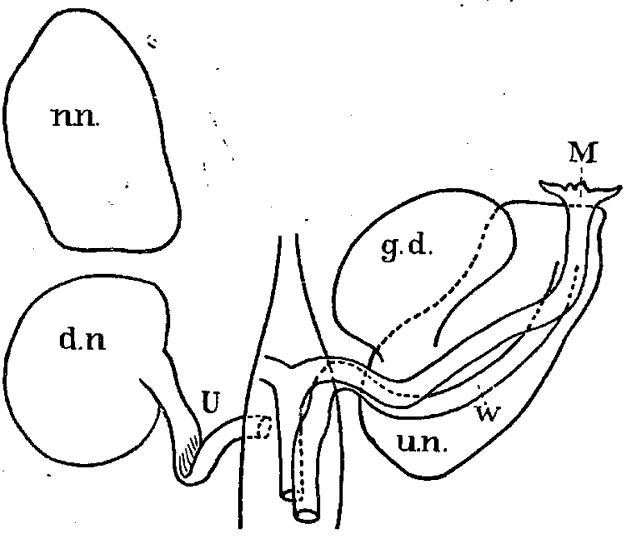

Abb. 1. Projektion der Organe des Urogenitalsystems auf der Frontalebene. n.n.: Nebenniere; d.n.: Definitive Niere; U.: Ureter; u.n.: Urniere; g.d.: Geschlechtsdrüse; W.: WOLFF'scher Gang; M.: MüLLER'scher Gang. Organ an seinem proximalen Pol treffen, ist keine Verbindung mit der Urniere mehr vorhanden, als stumpfer abgerundeter Fortsatz ragt dieser Pol frei in die Bauchhöhle nach oben. Beim Verfolgen der Schnitte in distaler Richtung tritt alsbald die Verbindung mit der Urniere auf, zunächst als Peritonealduplicatur, später als breitere Verbindung. Wir haben jetzt das Bild der in einen medialen und lateralen Anteil zerlegten Urogenitalfalte vor. uns, der mediale Streifen 
stellt die Plica genitalis, der laterale die Plica mesonephridica dar. Allmälich verliert sich in weiter nach unten gelegenen Schnitten die Abgrenznng der Plica genitalis, die Falte verstreicht immer mehr, während der Urnierenanteil mehr in den Vordergrund tritt. Es ist demnach nicht möglich genau den Schnitt zu bestimmen bis zu welchem die Geschlechtsdrüse sich nach unten erstreckt.

Das Geschlecht des Embryo lässt sich nicht mit Sicherheit bestimmen; das Verhalten der

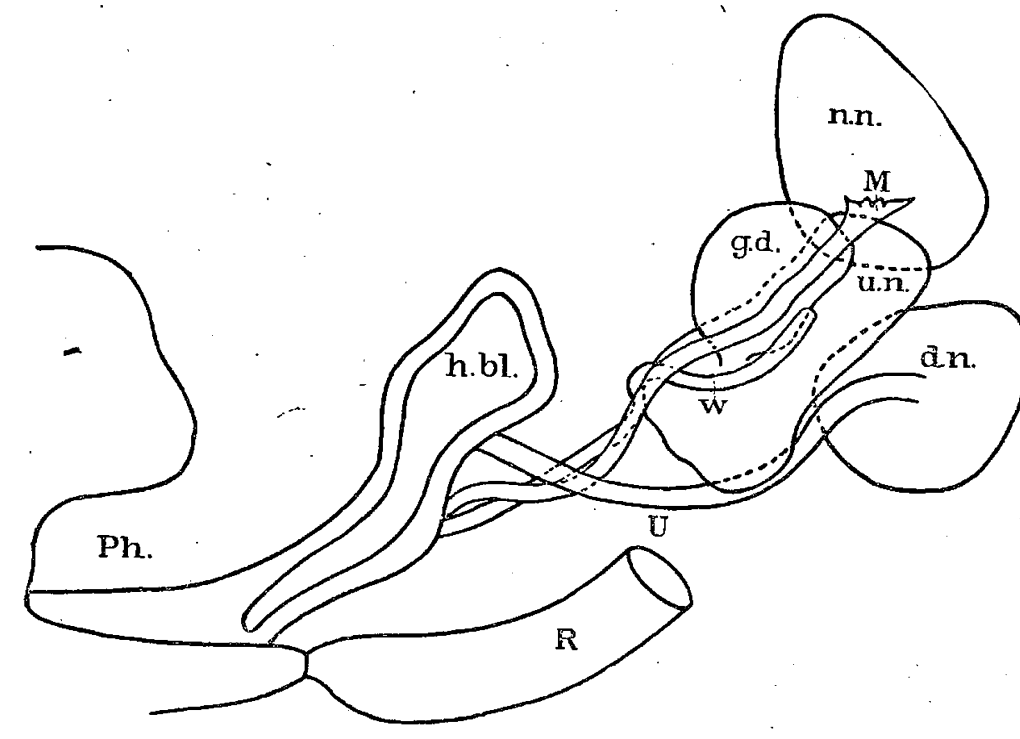

Abb. 2. Projektion der Organe des Urogenitalsystems auf der Sagittalebene. n.n.: Nebenniere; d.n.: Definitive Niere; U.: Ureter; u.n.: Urniere; g.d.: Geschlechtsdrüse; W.: WolfF'scher Gang: M.: MüLLER'scher Gang; R.: Rectum; h.bl.: Harnblase; ph.: Phallus. MüLLER'schen und WOLFF'schen Gänge bietet keine Anhaltspunkte; die ganze innere Organisation des Urogenitalapparates befindet sich noch in dem sogenannten indifferenten Zustand. Höchstens liesse sich aus dem Verhalten der äusseren Genitalien einiges ableiten, wenn auch hier die Schlussfolgerung, wegen Mangel an Vergleichsobjekten der selben Spezies, unsicher bleiben muss. Untersuchen wir die in diesem Gebiet vorhandenen Kennzeichen, so deuten sie auf das weibliche Geschlecht: Der Sulcus nympholabialis ist verhältnissmässig tief; die Pars phallica des Sinus urogenitalis ist nur hinten, in der Nähe des Anus offen; der Phallus ist im ganzen nach unten gekrümmt. Hervorzuheben ist, dass das Schnittbild der Geschlechtsdrüse keine

Merkmale verschafft, welche zur Bestimmung des Geschlechtes beitragen könnten.

\section{DIE EXTRAREGIONÄREN GESCHLECHTSZELLEN}

Die Ansicht, dass das Keimepithel, das heisst der Serosabezug der Plica genitalis, die alleinige Quelle der Geschlechtszellen sei, kann als aufgegeben betrachtet werden. Die Frage ob diese Stelle als Mutterboden für Geschlechtszellen überhaupt in Betracht kommt, respective aus welchen anderen Gebieten diese Zellen stammen, ist noch Gegenstand vielfacher Controverse; sie ist als nicht endgültig entschieden anzusehen. Bisher ist es noch für kein Wirbeltier gelungen eine ununterbrochene „Keimbahn" nachzuweisen, wie dieses bei Wirbellosen (Boveri bei Ascaris u. a.) möglich war. Die Literatur über diesen Gegenstand ist in den letzten Jahren stark angeschwollen, es würde uns viel zu weit führen auch nur das wichtigste hier anzuführen. Es seien hier nur kurz die Ergebnisse einer Untersuchung RUBASCHKIN's wiedergegeben; zur Einführung in das Thema, und weil unsere Beobachtungen sich diesen am besten angliedern lassen.

RUBASCHKIN stellte im Jahre 1908 das Vorkommen von extraregionären Geschlechtszellen bei Säugetieren fest ${ }^{1}$ ) und begründete auch ihre Qualifizieruing als solche. Er ging bei seiner Untersuchung von älteren Stadien aus; Kaninchen von 13 Tagen, Meerschweinchen und Katzen von 10 bis $11 \mathrm{~mm}$. wo Urgeschlechtszellen in der Geschlechtsdrüsenanlage zweifellos vorhanden waren und wo diese Zellen auch (fast ausnahmslos) alle nur an dieser Stelle aufzufinden sind. Bei jüngeren Embryonen, von etwa 5 bis $8 \mathrm{~mm}$. fand er die selben Gebilde im Mesenchym der benachbarten Teile, im Retroperitonealgebiet, in der Mesenterialwurzel. Und zwar war die Entfernung derselben von der Geschlechtsdrüsenanlage um so grösser, je jüngere Stadien untersucht wurden. Dabei zeigte as sich ausserdem, das topographisch ein Unterschied in dem Sinne vorhanden war, dass in mehr proximalen Teilen eines Embryo die Keimzellen weiter gewandert waren als in distalen desselben. Die Entwicklung war in

1) Rubaschkin, W. Zur Frage der Entstehung der Keimzellen bei Säugetierembryonen Anat. Anz. B. 32.1908. Rubaschkin, W. Ueber die Urgeschlechtszellen bei Säugetieren. Anat. Hefte. B. 39. 3. 1909. 
dieser Beziehung am cranialen Ende weiter fortgeschritten als am caudalen Ende; eine in der Ontogenese gewöhnliche Erscheinung.

Dass in der That eine Wanderung der betreffenden Zellen stattfindet, konnte er u. a. durch Zählungen in übereinstimmenden Körperbezirken bei verschiedenen Entwicklungsstadien nachweisen. Bei einem Caviaembryo von $8 \mathrm{~mm}$. fand er in der Höhe des $15^{\text {ten }}$ bis $17^{\text {ten }}$ Segmentes 46 Keimzellen, wovon $45 \mathrm{im}$ Gebiete der Geschlechtsdrüsenanlage; bei einem jüngeren Stadium $(6 \mathrm{~mm}$.) kamen in derselben Region 42 Zellen vor; von diesen lagen jedoch nicht weniger als 16 ausserhalb der Geschlechtsdrüse, hauptsächlich im Gekröse. Letztere Zellen haben demnach in der unmittelbar anschliessenden Entwicklungsphase den Weg bis zur Mesenterialwurzel, und von dort bis zu ihrem Endziel, der Keimdrüse zurückzulegen. Ob diese Wanderung eine passive, durch Wachstumsverschiebungen hervorgerufene, oder eine active, amöboide, ist, bleibe dahingestellt. Nach RuBASCHKIN trifft beides zu.

In noch jüngeren Stadien (Kaninchen und Meerschweinchen von 22 Segmenten) schliesslich, traf RUBASChKIN die Keimzellen im Darmentoderm, und in der unmittelbaren Umgebung desselben; manche waren im Moment ihrer Emigration fixiert worden, sodass der Zellleib halbwegs zwischen den Epithelzellen des Darmes, halbwegs ausserhalb derselben hervorragend, gefunden wurde. Auch hier liess sich wieder feststellen, dass die emigrierten Zellen in grösserer Anzahl weiter proximal, die noch im Entoderm, sich befindenden Zellen in grösserer Anzahl weiter distal aufzufinden waren; dieselbe Erscheinung welche oben erwähnt wurde. Anschliessend an diese Befunde traf RuBAschkin bei Embryonen von 17 bis 7 Segmenten Zustände wo die Keimzellen nur im Entoderm, und zwar im caudalen Abschnitt desselben, sich nachweisen liessen. Bei einigen dieser jüngsten Stadien waren sie in der noch nicht differenzierten Region, welche der caudalen Fortsetzung des Primitivstreifens entspricht, aufzufinden.

Zusammenfassend können wir demnach feststellen dass aus RUBASCHKIN's Untersuchungen hervorgeht dass Urgeschlechtszellen bei verschiedenen Säugern während der Ontogenese eine Wanderung durchmachen, wobei sie zunächst im çaudalen Abschnitt des Entoderm aufgenommen werden. Sie verbleiben hier nur kurz, verlassen das Epithel und wandern zwischen den Mesenchymzellen des Gekröses dorsalwärts bis an dessen Wurzel, um von dort aus den WolfF'schen Körper und schliesslich ihr Endziel, die Anlage der Geschlechtsdrüse zu erreichen.

Die Tatsache der Wanderung der Urgeschlechtszellen in der eben referierten Weise ist auch bei einigen anderen Säugern und beim Menschen festgestellt worden ${ }^{1}$ ). Ueber das weitere Schicksal derselben, ob aus ihnen in der That die definitiven Geschlechtsprodukte hervorgehen, hat sich noch keine einheitliche Auffassung durchringen können. Neben der extremen Meinung einerseits dass sie die alleinige Quelle aller zur Reifung gelangender Geschlechtszellen sind (RubaschKIN, SwIFT) steht andererseits die Auffassung, es handele sich bei den Wanderzellen überhaupt nicht um Elemente welche mit der Fortpflanzung irgend etwas zu thun hätten; es seien lediglich zeitweilig hypertrophische Zellen welche später verschwinden (v. BerEnberG-Gossler, ${ }^{2}$ ). Zwischen diesen Extremen nehmen andere $\left(\right.$ FIRKET ${ }^{3}$ ) eine vermittelnde Stellung ein: sie erkennen die Wanderzellen als Geschlechtszellen an, nehmen aber an, dass alle, oder doch die überwiegende Mehrzal derselben, degenerieren und somit an der Entwicklung der zur Reifung gelangenden Eier und Spermien nicht beteiligt sind. Letztere sollten dann vom Keimepithel ihren Ursprung nehmen (regionäre Geschlechtszellen). Aus allerletzter Zeit ist schliesslich zu erwähnen, dass die schon früher geäusserte Ansicht, einige Arten von Tumoren (Teratome, Embryome) seien auf Wucherung versprengter oder zurückgebliebener Wanderzellen zurückzuführen, in RotTER ${ }^{4}$ ), neuerdings einen Vertreter gefunden hat, welcher sogar sämtliche malignen Neubildungen, Sarcome, Carcinome, von derartigen Elementen glaubt herleiten zu müssen.

Die Reihe wichtiger Fragen, welche sich an das Problem der extraregionären Geschlechtszellen knüpft, ist hiermit keineswegs erschöpft, es war hier nur darum zu tun kurz einige derselben anzudeuten. Betrachten wir nun, wie sich die fraglichen Elemente bei unserem jugendlichen Faultier ver-

1) Fuss, A. Ueber die Geschlechtszellen des Menschen und der Säugetiere. Arch. f. Micr. Anat. B. 81. II 1912 Fuss, A. Ueber extra regionäre Geschlechtszellen bei einem menschlichen Embryo von 4 Wochen. Anat. Anz. B. 39.1911.

2) V. Berenberg-Gossler, H. Die Urgeschlechtszellen des Hühnerembryo's etc. Arch. f. Micr. Anat. B. 81. Ir 1912.

3) Firket, J. Recherches sur l'organogénèse des glandes sexuelles chez les oiseaux. I et II. Arch. de Biol. T. 29, 30.

4) ROTTER, H. Histogenese der malignen Geschwülste. Zeitschr. f. Krebsforschung. Bd. 18. 3. 1921. 
halten. Der Embryo von Bradypus, welcher der vorliegenden Untersuchung zu grunde liegt, zeigt in verschiedenen Körperbezirken eigenartige Zellen, welche, bezugnehmend auf die Ergebnisse der oben referierten Arbeiten, als extraregionäre Geschlechtszellen aufzufassen sind. Sie bilden eine Stütze für die Anschauung, dass das Entoderm an der Lieferung dieser Zellen mitbeteiligt ist, und zeigen, dass bei diesem Säugetier die Wanderung dieser Zellen bis in ein verhältnismässig spätes Stadium andauert.

Wie in der Einleitung geschildert, ist die Differenzierung des ganzen Urogenitalapparates weit fortgeschritten, das bemerkenswerte ist, dass trotzdem auch jetzt noch die Migration von Zellen aus dem Entoderm, durch das Mesenterium, durch das Urnieren- und Geschlechtsdrüsenligament, sich nachweisen lässt. Sie zeichnen sich vor allem durch ihre Grösse von den umgebenden Elementen aus; diese beträgt etwa $17 \mu$, die Kerngrösse 10 bis $13 \mu$. Beim Menschen fand Fuss als grössten Kerndurchmesser $12^{3} /_{4} \mu$, als grössten Protoplasmadurchmesser $19^{1}{ }_{2} \mu$. In den mit Hämatoxylin-Eosin gefärbten Schnitten nimmt der Kern eine ziemlich hellblaue Farbe an, ein oder zwei dunkel gefärbte Kernkörperchen sind vorhanden, das Protoplasma ist blassrot. In den Geschlechtsdrüsen finden sich Elemente wie die hier beschriebenen in grosser Anzahl; diejenigen welche noch im Entoderm, oder auf dem Wege vom Entoderm zur Geschlechtsdrüse angetroffen werden, sind wohl als Nachzügler zu betrachten; in jüngeren Stadien hat wohl die grosse. Mehrheit der extra-regionären Zellen ihre Wanderung unternommen. $\mathrm{Ob}$ all diese Elemente, welche wir in der Geschlechtsdrüsenanlage antreffen, durch Einwanderung dahingelangt sind, muss hier unentschieden bleiben.

Wie oben erwähnt ragt der proximale Pol der Keimdrüse frei in die Bauchhöhle nach oben; in dieser Region ist die Einwanderung abgeschlossen. Urgeschlechtszellen fiinden sich in grosser Anzahl auf der ganzen Oberfläche des Schnittbildes der Geschlechtsdrüsenanlage zerstreut. Viele weisen einen grossen, mit pseudopodienähnlichen Ausläufern versehenen Protoplasmakörper auf.

Verfolgen wir die Schnittbilder in caudaler Richtung, so treffen wir alsbald auf die Verbindung von Keimdrüse und Urniere, und zugleich verschiebt sich die Befestigungsstelle des Urnierenligamentes an die dorsale Rumpfwand in medialer Richtung, sodas sie sich der Radix mesenterii nähert. Hier treten nun die Wanderzellen in verhältnismässig grosser Anzahl auf, fast jeder Schnitt zeigt einen oder

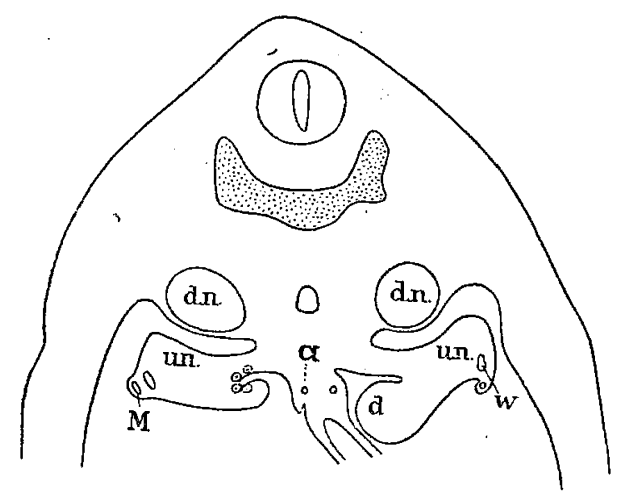

Abb. 3. Querschnitt des Rumpfes; hintere Rumpfwand. Im Bild links vier Keimzellen schematisiert wiedergegeben. d.n.: Definitive Niere; u.n.: Unniere; M.: MULLERscher Gang W.: WoLfFscher Gang; d.: Geschlechtsdrüse; a.: Arterie der Gekrösewurzel.mehrere derselben. Und zwar trifft man sie: 1 nahe der Keimdrüse im Urnierenligament, 2 in der dorsalen Pumpfwand im Areal zwischen Radix mesenterii und Anheftungsstelle des Urnierenligamentes, 3 im Wurzelgebiet des Gekröses, 4 in den dorsalen Partien des Gekröses selbst. Sie liegen zwischen den Mesenchymzellen, meistens einzeln, manchmal zwei nebeneinander, selten mehrere zusammen. Letzteren Zustand beobachtet man einige Male im Urnierenligament, wo Reihen von vier oder fünf Zelien angetroffen werden. Kernteilungsfiguren, welche in den somatischen Zellen in grosser Anzahl vorhanden sind, konnten in den Wanderzellen kein einziges Mal mit Sicherheit erkannt werden; wohl machen einige Kerne den Eindruck des Zerfalles, der Degeneration. Im dorsalen Gebiet des Gekröses liegt rechts und links eine Arterie, von zwei Venen begleitet; in der Nähe dieser Gefässe, wo das umgebende Mesenchym compactere Structur aufweist, findet man nicht selten Exemplare der gesuchten Zellen. Es macht den Eindruck als ob sie hier gewissermassen eingefangen, oder doch wenigstens durch das dichtere Mesenchym aufgehalten und an ihrer Wanderung behindert wurden.

Einige Abbildungen mögen das geschilderte Verhalten illustrieren: so zeigt uns Abbildung 3 einen Schnitt (34.3.4.) nahe dem cranialen Ende von Urniere und Keimdrüsenanlage, welcher an der medialen Seite des Urnierenligamentes eine Reihe von vier Wanderzellen enthält. In ihrer Aufeinanderfolge deuten sie so zu sagen die Richtung ihrer Fortbewegung an. Dasselbe Bild zeigt uns die Lage der beiden Arterien im dorsalen Gekröseabschnitt, wovon oben die Rede war. Eine derselben ist in Abbildung 4 bei stärkerer Vergrösserung wiedergegeben; die Figur entstammt einem anderen Schnitt. In der Nähe des Gefässes liegen zwei Urgeschlechtszellen im dichteren Mesenchym dieses Gebietes. Ein ähnliches Verhalten lässt sich in der Umgebung dieser Gefässe des öfteren feststellen. Dass die 
Wanderzellen auch im Gebiet zwischen den heiden abgebildeten Stellen, im Befestigungsareal des Gekröses, und in der dorsalen Rumpfwand angetroffen werden, wurde oben erwähnt. Eine Regel, wonach in mehr proximalen Schnitten die Zellen näher der Keimdrüse, in mehr distalen in grösserer Anzahl im Mesenterium anzutreffen wären, lässt sich aus den Schnittbildern nicht ableiten. In unregel-

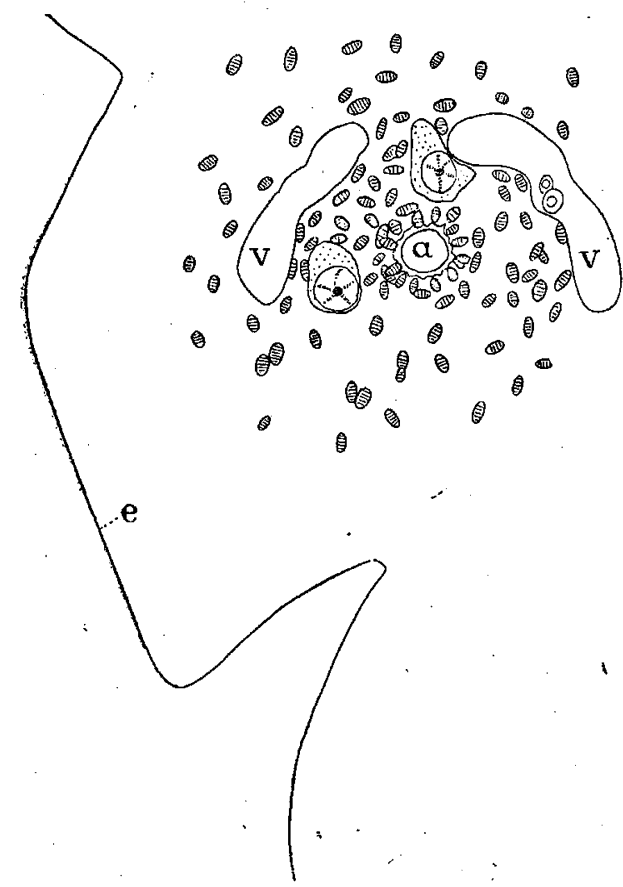

Abb. 4. Zwei Keimzellen in der Nähe der Arterie (a); vv.: Venen; e.: Epithelbezug der Serosa. mässiger Folge tauchen sie an verschiedenen Stellen ihrer Bahn auf. Denken wir an die oben erwähnten Befunde von Urgeschlechtszellen im Entoderm des Darmkanales, so liegt es nahe, auch hier in diesem Gebiete nach ihnen $\mathrm{zu}$ suchen. Von vorneherein erscheint die Wahrscheinlichkeit sie bei dem weit vorgeschrittenem Entwicklungsstadium, welches vorliegt, noch an dieser Stelle zu treffen, gering. Der Darm weist bereits ein erhebliches Längenwachstum auf und hat eine ziemlich ansehnliche Musculatur, hauptsächlich zirculärer Natur, ausgebildet. Am ehesten wäre zu erwarten, dass sie in caudalen Anteilungen des Darmrohres anzutreffen wären, war doch hier bei den jungen Stadien anderer Spezies len sich verbreiteten. In der That weist des tangential angeschnittedas Epithel des künftigen Rectum und nen Darmrohres. m.: MusDickdarm die gesuchten Elemente in Spaltraum.

gar nicht geringer Anzahl auf. Durch

ihre Kerngrösse lassen sie sich leicht und sicher von den umgebenden Entodermzellen unterscheiden, ihr Habitus stimmt mit den oben beschriebenen, an anderen Orten gefundenen Keimzellen vollkommen überein. Am deutlichsten sind sie auf Tangentialschnitten des gekrümmten Darmrohres zu erkennen. Abbildung 5 (34. 1.5.) giebt ein Bild einer derartigen, im Entoderm zurückgebliebenen Urgeschlechtszelle. Ob diese Elemente, wo durch die Ausbildung der Muskulatur ein Hinderniss für ihre Fortbewegung geschaffen wurde, noch im Stande wären ihr Endziel zu erreichen, scheint wenig wahrscheinlich. Fraglich bleibt was in diesem Falle ihr weiteres Schicksal wäre. Auffallend ist es jedenfalls, dass nur sehr vereinzelt Wanderzellen in der unmittelbaren Nähe des Darmrohres, ausserhalb der Muskulatur angetroffen werden. Dieses spricht dafür, dass die Muskelschicht als Hemmnis für ihren Austritt aus dem Entoderm eine Rolle spielt, und das wir das letzte Stadium des Migrationsvorganges bei diesem Embryo vor uns haben. 